\title{
RXTE monitoring of Centaurus A
}

\author{
S. Benlloch ${ }^{1}$, R. E. Rothschild ${ }^{2}$, J. Wilms ${ }^{1}$, C. S. Reynolds ${ }^{3,4}$, W. A. Heindl ${ }^{2}$, and R. Staubert ${ }^{1}$ \\ 1 Institut für Astronomie und Astrophysik - Astronomie, University of Tübingen, Waldhäuser Str. 64, \\ 72076 Tübingen, Germany \\ 2 Center for Astrophysics and Space Sciences, University of California at San Diego, La Jolla, CA 92093, USA \\ 3 JILA, University of Colorado, Boulder, CO 80309-0440, USA \\ 4 Hubble Fellow
}

Received 2 January 2001 / Accepted 21 March 2001

\begin{abstract}
We report on the analysis from $\sim 110 \mathrm{ks}$ of X-ray observations of Centaurus A carried out with the Proportional Counter Array (PCA) and the High Energy X-ray Timing Experiment (HEXTE) instruments on Rossi X-ray Timing Explorer (RXTE) during three monitoring campaigns over the last 4 years (10 ks in $1996,74 \mathrm{ks}$ in 1998, and $25 \mathrm{ks}$ in 2000). The joint PCA/HEXTE X-ray spectrum can be well described by a heavily absorbed power law with photon index 1.8 and a narrow iron line due to fluorescence of cold matter. The measured column depth decreased by about 30\% between 1996 and 2000, while the detected 2-10 keV continuum flux remained constant between 1996 and 1998, but increased by 60\% in 2000. Since in all three observations the iron line flux did not vary, a corresponding decrease in equivalent width was noted. No appreciable evidence for a reflection continuum in the spectrum was detected. We present the interpretation of the iron line strength through Monte Carlo computations of various geometries. No significant temporal variability was found in Cen A at time scales from days to tens of minutes.
\end{abstract}

Key words. galaxies: active - galaxies: individual (Cen A) - X-rays: general - X-rays: galaxies

\section{Introduction}

At a distance of 3.5 Mpc (Hui et al. 1993), Centaurus A (NGC 5128) is by far the nearest active galactic nucleus (AGN). Cen A is a giant double-lobed radio source usually classified as a low luminosity Fanaroff \& Riley (1974) Class I radio galaxy, a misdirected BL Lac object (Morganti et al. 1992), a Seyfert 2 object (Dermer \& Gehrels 1995), and a radio-loud AGN viewed from the side $\left(\sim 70^{\circ}\right)$ of the jet axis (Ebneter \& Balick 1983). Even though the nucleus is heavily obscured (in part by the famous dust-lane that is seen to cross the galaxy), Cen A is one of the brightest extragalactic X-ray sources in the sky and the only AGN where a high quality spectrum from $0.5 \mathrm{keV}$ to $1 \mathrm{GeV}$ can be obtained (Steinle et al. 1998). This makes it a valuable laboratory to test our understanding of the high-energy processes occurring within AGN. A recent comprehensive review of Cen A is given by Israel (1998).

Previous observations have shown Cen A to have highly complex X-ray/gamma-ray properties (Turner et al. 1997a; Döbereiner et al. 1996; Feigelson et al. 1981; Schreier et al. 1979) with multi-temperature diffuse flux, a spatially resolved jet in radio and X-rays (Kraft et al.

Send offprint requests to: S. Benlloch, e-mail: benlloch@astro. uni-tuebingen.de
2000; Morganti et al. 1999), and a nuclear component with complex low energy absorption and a photon index of $\sim 1.7$. Iron K-shell absorption and fluorescent line emission at $\sim 6.4 \mathrm{keV}$ with an equivalent width of $\sim 100 \mathrm{eV}$ have been observed (Turner et al. 1997a). One unusual spectral feature of Cen A is that there is no evidence for a strong Compton reflection component (Woźniak et al. 1998 , found $\Omega / 2 \pi<0.15$ ). This implies there is little cold, Thomson-thick material in the close vicinity of the AGN that reflects X-rays into our line of sight. Taken together with the iron line equivalent width, this result is consistent with transmission of the primary flux through the line-ofsight absorber and viewing the accretion disk nearly edgeon. On the other hand, the lack of a reflection component is at odds with the "Unified Model of AGN" (e.g., Krolik 1999, Ch. 12), where Seyfert galaxies do show significant reflection components (Smith \& Done 1996).

In this work we present results of three observations performed by the Rossi X-ray Timing Explorer (RXTE) - one of $10 \mathrm{ks}$ in 1996 August, one of 74 ks in 1998 August and one of $25 \mathrm{ks}$ in 2000 January. Preliminary results from the 1996 August observation, using earlier versions of the response matrix and background models, have been presented by Rothschild et al. (1999). Here we re-analyze these data using the new and improved PCA response matrices and background models, and present results on all 


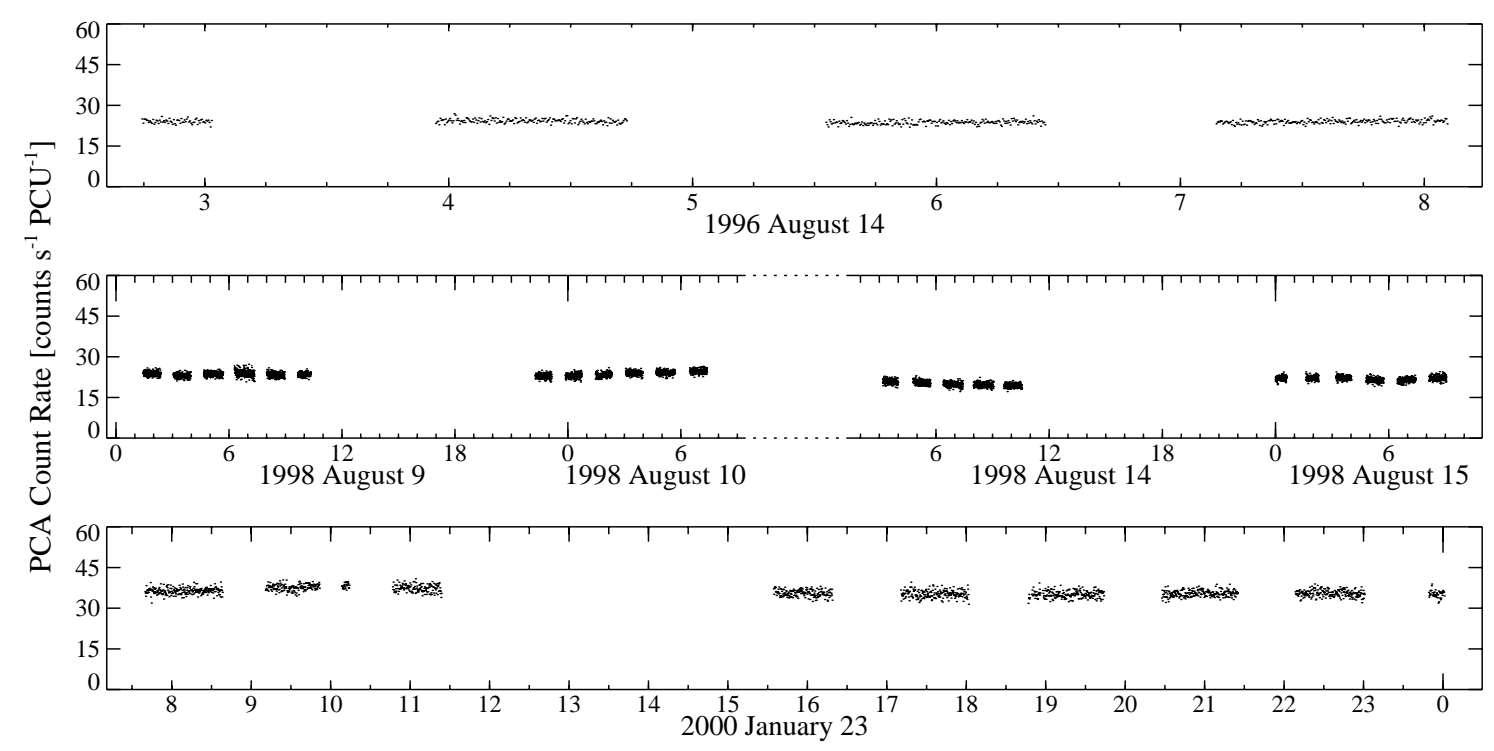

Fig. 1. Background-subtracted PCA-RXTE light curves of Cen A for the three RXTE monitoring campaigns in the years 1996 (upper panel), 1998 (middle panel) and 2000 (lower panel). The data have a resolution of $16 \mathrm{~s}$ and are plotted versus Universal Time (UT) in hours on the given date

Table 1. Details of the RXTE monitoring campaigns of Cen A

\begin{tabular}{cccc}
\hline Numb. & $\begin{array}{c}\text { Obs. Date } \\
(\text { ymd })\end{array}$ & $\begin{array}{c}\text { Exposure } \\
(\mathrm{s})\end{array}$ & $\begin{array}{c}\text { Count Rate } \\
\left(\text { counts s }^{-1}\right.\end{array}$ PCU $\left.^{-1}\right)$ \\
\hline \hline 1. & 1996, Aug. 14 & 10528 & 24 \\
2. & 1998, Aug. 9-15 & 73984 & 22 \\
3. & 2000, Jan. 23 & 24880 & 36 \\
\multicolumn{3}{c}{ total $~ 109.5 \mathrm{ks}$} \\
\hline
\end{tabular}

(The count rate is the mean of the $2-20 \mathrm{keV}$ PCA background subtracted count rate).

three observations. The remainder of this paper is structured as follows. In Sect. 2 we give the details of the observations and our data analysis procedure. Section 3 presents the results from the spectral analysis and the timing analysis. In Sect. 4 we discuss our results and summarize the paper.

\section{Observations and data reduction}

There are two pointed instruments on-board RXTE, the Proportional Counter Array (PCA) and the High Energy X-ray Timing Experiment (HEXTE). RXTE observed Cen A for a total of $\sim 110 \mathrm{ks}$ over the last five years. An observation log is given in Table 1. The PCA light curves are shown in Fig. 1.

The PCA consists of a set of five co-aligned xenon/methane (with an upper propane layer) proportional counter units (PCUs) with a total effective area of $\sim 7000 \mathrm{~cm}^{2}$. The instrument is sensitive in the energy range from $2 \mathrm{keV}$ to $\sim 100 \mathrm{keV}$ (Jahoda et al. 1997). As the response matrix is best calibrated between $\sim 2$ and $20 \mathrm{keV}$, we will restrict our analysis to this energy range. To in- crease the signal to noise ratio we chose only the data from the top xenon layer. Background subtraction of the PCA data was performed using the "faint source model". To reduce the uncertainty of the PCA background model, we ignore data measured in the $30 \mathrm{~min}$ after South Atlantic Anomaly (SAA) passages. Furthermore, data were not accumulated at times of high electron contamination as expressed by a certain ratio of veto rates in the detectors, the so-called "electron ratio". We excluded times during which the "electron ratio" was larger than 0.1 in at least one of the detectors. We accounted for the remaining uncertainty in detector calibrations by including a systematic error of $1 \%$ in the data. See Wilms et al. (1999) for an in-depth discussion of the PCA calibration issues.

HEXTE consists of two clusters of four $\mathrm{NaI}(\mathrm{TI}) / \mathrm{CsI}(\mathrm{Na})$ phoswich scintillation counters that are sensitive in the range $15-250 \mathrm{keV}$ (Rothschild et al. 1998). Its total effective area is $\sim 1600 \mathrm{~cm}^{2}$. Background subtraction is done by sequential rocking of the two clusters on and off of the source position to provide a direct measurement of the background during the observation. Thus, no calculated background model is required. HEXTE data between $20 \mathrm{keV}$ and $200 \mathrm{keV}$ were used. To ensure good statistical accuracy the spectrum was rebinned for fitting, and no systematic errors were incorporated in the HEXTE data.

The standard data from both instruments were used for the accumulation of spectra and light curves with $16 \mathrm{~s}$ time resolution. To extract spectra and light curves, we used the RXTE analysis software in FTOOLS 5.0. During part of the observations some PCUs were turned off, and we took this into account when we added all good PCU data together and combined all background spectra. The total PCA response matrix was the average of the respective estimates for each individual detector combination, 
Table 2. Best fit spectral parameters for the 1996, 1998 and 2000 data set fitted separately (Separated spectra) and for the three data sets fitted simultaneously (Joined spectrum)

\begin{tabular}{|c|c|c|c|c|c|c|c|c|c|c|c|}
\hline & & \multicolumn{3}{|c|}{ Iron Line } & \multicolumn{2}{|c|}{ Power law } & \multicolumn{2}{|c|}{ Detector normalization } & \multirow{2}{*}{$\begin{array}{c}F_{(2-10 \mathrm{keV})} \\
\quad \times 10^{-2}\end{array}$} & \multirow[t]{2}{*}{$\chi_{\text {red }}^{2}$} & \multirow[t]{2}{*}{ d.o.f } \\
\hline & $\times 10^{22} \mathrm{~cm}^{-2}$ & $\begin{array}{l}E_{\mathrm{Fe}} \\
\mathrm{keV}\end{array}$ & $\begin{array}{c}A_{\text {Line }} \\
\times 10^{-4}\end{array}$ & $\begin{array}{c}E W \\
\mathrm{eV}\end{array}$ & $\Gamma$ & $\begin{array}{c}A_{\mathrm{PL}} \\
\times 10^{-2}\end{array}$ & $A_{\text {Det }_{\mathrm{A}}}$ & $A_{\operatorname{Det}_{\mathrm{B}}}$ & & & \\
\hline \multicolumn{12}{|c|}{ Separated spectra } \\
\hline 1996 & $11.30(14)$ & $6.56(16)$ & $4.36(74)$ & $144(26)$ & $1.92(15)$ & $0.135(2)$ & $0.90(6)$ & $0.88(9)$ & $2.28(4)$ & 0.75 & 64 \\
\hline 1998 & $9.41(28)$ & $6.47(8)$ & $3.97(63)$ & $142(27)$ & $1.90(2)$ & $0.114(4)$ & $0.92(3)$ & $0.90(3)$ & $2.21(4)$ & 0.51 & 77 \\
\hline 2000 & $7.87(27)$ & $6.27(9)$ & $5.46(98)$ & $120(26)$ & $1.82(2)$ & $0.150(6)$ & $0.92(3)$ & $0.92(3)$ & $3.60(6)$ & 0.40 & 60 \\
\hline \multicolumn{12}{|c|}{ Joined spectrum } \\
\hline 1996 & $10.66(18)$ & $6.49(7)$ & $5.34(62)$ & $177(24)$ & $1.88(1)$ & $0.122(3)$ & 0.90 & 0.88 & $2.28(3)$ & 0.78 & 209 \\
\hline 1998 & $9.15(19)$ & $6.45(8)$ & $4.28(54)$ & $154(22)$ & & $0.108(2)$ & 0.92 & 0.90 & $2.21(3)$ & & \\
\hline 2000 & $8.70(19)$ & $6.29(11)$ & $3.82(87)$ & $82(20)$ & & $0.173(4)$ & 0.92 & 0.92 & $3.58(3)$ & & \\
\hline
\end{tabular}

$E_{\mathrm{Fe}}$ : line energy. $A_{\text {Line }}$ : Line normalization $\left(\mathrm{ph} \mathrm{cm}^{-2} \mathrm{~s}^{-1}\right.$ in the line). The Gaussian line width was fixed at $\sigma=0.2 \mathrm{keV} . E W$ : line equivalent width. $\Gamma$ : Photon index of the power law. $A_{\mathrm{PL}}$ : Power law norm $\left(\mathrm{ph} \mathrm{keV}^{-1} \mathrm{~cm}^{-2} \mathrm{~s}^{-1}\right.$ at $\left.1 \mathrm{keV}\right) . F_{(2-10 \mathrm{keV})}$ : Flux at the energy range of 2 to $10 \mathrm{keV}$ in units of $\mathrm{ph} \mathrm{cm}^{-2} \mathrm{~s}^{-1} \cdot A_{\text {Det }_{\mathrm{A}}}$ : Ratio of HEXTE to PCA Normalization for Cluster A. $A_{\text {Det }_{\mathrm{B}}}$ : Ratio of HEXTE to PCA Normalization for Cluster B. The uncertainties are $90 \%$ for one parameter $\left(\Delta \chi^{2}=2.7\right)$, and are shown in units of the last digit shown. Parameters in italics were frozen.

weighted by the fraction of photons measured during the time that such combination was active. For HEXTE we used the standard response matrices dated 1997 March 20, treating each cluster individually in the data analysis.

On-board RXTE there is a third scientific instrument, the All-Sky Monitor (Levine et al. 1996). The ASM is composed of three scanning shadow cameras, each of which is a position sensitive xenon proportional counter that views a $6^{\circ} \times 90^{\circ} \mathrm{FWHM}$ section of sky through a one-dimensional coded mask. Each camera has a net active area for detecting X-rays of $\sim 30 \mathrm{~cm}^{2}$. It surveys about $80 \%$ of the sky $5-10$ times each day for $\sim 100 \mathrm{~s}$. The overall energy range of the ASM is $1.5-12 \mathrm{keV}$ which is telemetered in three energy channels $(1.3-3.0 \mathrm{keV}, 3.0-5.0 \mathrm{keV}$, and $5.0-$ $12.2 \mathrm{keV})$. Figure $4 \mathrm{~b}$ shows the RXTE ASM counting rate for Cen A from the beginning of the RXTE mission, rebinned in $14 \mathrm{~d}$ bins; the arrows denote the times when the PCA/HEXTE monitoring campaigns were made. We used the "Data by dwell quick-look results" provided by the RXTE ASM team, available from MIT ASM Light Curve Overview Webpages. We took only flux solutions for which $\chi_{\text {red }}^{2}<1.2$, with a background estimated between 1 and 10 counts $^{-1}$ (Levine et al. 2000), and with a full dwell-exposure time (i.e., $90 \mathrm{~s}$ ) .

Also shown in Fig. 4c are the $20-100 \mathrm{keV}$ count rates from the BATSE instrument on board the Compton Gamma Ray Observatory (CGRO) averaged over two week intervals from 1991-1999 (data provided by C. Wilson, priv. comm.). BATSE consists of eight identical $\mathrm{NaI}(\mathrm{Tl})$ scintillation detector modules located at the corners of CGRO. Sources are detected using the Earth occultation technique. During each CGRO orbit, the Earth is seen by BATSE to sweep across a band in the sky extending $\sim 35^{\circ}$ above and below the orbit plane. As each source enters into (exits from) occultation by the Earth, count rates in the source-facing detectors decreases (increase) according to the source intensity.

\section{Data analysis}

\subsection{Spectral analysis}

The PCA and HEXTE data were fit simultaneously using XSPEC v. 11.0q (Arnaud 1996). The spectrum was modelled by a heavily absorbed power law plus a Gaussian emission line model, of the form

$$
\begin{aligned}
N_{\mathrm{ph}}(E)= & A_{\text {Det }}\left[\mathrm{e}^{-\sigma_{\mathrm{ISM}}(E) N_{\mathrm{H}}} A_{\mathrm{PL}} E^{-\Gamma}\right. \\
& \left.+\frac{A_{\text {Line }}}{\sqrt{2 \pi \sigma^{2}}} \mathrm{e}^{-\frac{\left(E-E_{\mathrm{Fe}}\right)^{2}}{2 \sigma^{2}}}\right]
\end{aligned}
$$

To account for the uncertainty in the effective areas of the three detector systems (the PCA and the two HEXTE clusters), we included their relative normalizations, $A_{\text {Det }}$, as a fit parameter, setting the PCA normalization to unity. For the photoelectric absorption of the interstellar medium, $\sigma_{\text {ISM }}$, we used the photo-electric absorption cross section of Morrison \& McCammon (1983) where $N_{\mathrm{H}}$ is the equivalent hydrogen column. The fit parameters of the photon power law are $\Gamma$ the photon index and $A_{\mathrm{PL}}$ the power law norm $\left(\mathrm{ph} \mathrm{keV}^{-1} \mathrm{~cm}^{-2} \mathrm{~s}^{-1}\right.$ at $\left.1 \mathrm{keV}\right)$. To model the iron line component a Gaussian line profile was added where $E_{\mathrm{Fe}}$ is the line energy, $A_{\text {Line }}$ the total $\mathrm{ph} \mathrm{cm}^{-2} \mathrm{~s}^{-1}$ in the line, and $\sigma$ the Gaussian line width. As preliminary fits with the iron line width as a free parameter resulted in widths narrower than the spectral resolution of the PCA, we fixed the line width to $\sigma=0.2 \mathrm{keV}$. 
The spectral model was fitted to each data set separately (the 1996, 1998, and 2000 separated spectra) and to the three data sets simultaneously (the joined spectrum). Table 2 provides fitted parameters and their associated uncertainties. Our adopted simple spectral model (Eq. (1)) provides a fully acceptable fit in each case. Note that we assume that the iron line is not absorbed by the intervening material. This physically implies that the reprocessing region in which the $\mathrm{Fe}$ line is produced is outside the absorbing line of sight, and thus, the high absorption column is very near the X-ray emission region at the core of the AGN. This assumption only affects the inferred flux of the line and its computed equivalent width.

For all three observations, the energy of the iron line, $\sim 6.4 \mathrm{keV}$, indicates that the emission is from cool, i.e., at most weakly ionized material. It is either possible, 1) that the Fe-line emitting material lies directly along our line of sight, and could thus be associated with matter producing the observed $N_{\mathrm{H}}$, or 2) it is possible that the Fe-line emitting material is caused by fluorescent emission of a larger spatial region. In the former case, i.e., the emission of line photons from within our line of sight, we would expect the line flux to be correlated with $N_{\mathrm{H}}$. Within the uncertainty of the fit parameters, such a correlation is not found, as there is no variability in the line flux with observation while $N_{\mathrm{H}}$ varies. Such a constancy of the line flux is only possible if the line emission originates outside of our line of sight, for example within the torus. Here, the line strength would not correlate with the variation of $N_{\mathrm{H}}$. Furthermore, we would also not expect a correlation with changes of the source flux since these would be smeared out on the light travel timescale, which can be several years. Note that the equivalent width of the line decreases between the observations. This decrease, however, is purely due to the factor of 1.6 increase in the continuum flux over the observations. Finally, we note that our conclusion, that the Fe-line emitting material lies outside of our direct line of sight, is consistent with estimates for the line strength caused by the absorbing material alone, which predict that $<20 \%$ of the line flux is caused by fluorescence in the absorbing material (see Sect. 4).

Apart from the changes in the power law normalization $(+11 \%$ with respect to 1996$)$ and in the $N_{\mathrm{H}}(-30 \%$ with respect to 1996) there is no significant changes of the spectral parameters between the three observations. In passing, we note that the same holds also true when analyzing the spectra observed during each individual orbit. No significant variation of the spectral parameters was found within each monitoring observation.

As the spectral parameters remain roughly constant between the observations, it is possible to further constrain the shape of the spectral continuum by fitting all data simultaneously. We first attempted to fit all the data with a single model by coupling all spectral parameters (with the exception of the power law flux) for the three data sets. Our fit to this model had strong residuals in the region below $10 \mathrm{keV}$ (see Fig. 2c), clearly indicating the variation in $N_{\mathrm{H}}$. The fit corroborates that the shape of the underlying power-law continuum might be constant. We therefore modeled the combined spectrum using a model where all parameters were allowed to vary freely with the exception of the photon index, which was held fixed for the three data sets. The fitted parameters for this fit are listed in Table 2 (joined spectrum), a plot of the spectral model and its residuals is shown in Figs. 2a and 2b. The good $\chi^{2}$ of this fit indicates that $\Gamma$ can indeed be assumed to be constant for the three observations.

In order to test for the presence of a Compton reflection component, a power law plus Compton component (XSPEC model pexrav, Magdziarz \& Zdziarski 1995) fit was performed to both, the individual observations and the joined data with a single power law index. The $2 \sigma$ upper limit for the reflection component, $\Omega / 2 \pi$, expressed as the ratio of the solid angle for primary flux scattered into our line of sight to an infinite slab, was found to be 0.09 , $0.09,0.08$, and 0.05 for observations $1,2,3$, and joined, respectively, assuming an inclination angle of $70^{\circ}$. This result clearly implies that no significant reflection features are present, confirming the earlier OSSE and RXTE results (Rothschild et al. 1999; Kinzer et al. 1995).

We also tested the RXTE data for the presence of a spectral break at higher energies, using an exponentially cutoff model (XSPEC model cutoffpl). The best fit model gave a cutoff-energy with a lower limit of $500 \mathrm{keV}$ and provides an equally adequate fit to the data as the straight power law $\left(\chi_{\text {red }}^{2}=0.65\right)$. This result is consistent with CGRO OSSE data, where a cutoff energy of $300 \mathrm{keV}$ or higher was found (Kinzer et al. 1995). We would not be sensitive to a break of $\Delta \Gamma=0.5$ at $150 \mathrm{keV}$, as reported by Steinle et al. (1998), due to limited statistics above $150 \mathrm{keV}$.

To summarize, the RXTE spectrum of Cen A can be best described by a heavily absorbed power law extending above $200 \mathrm{keV}$ and an iron line from cold matter. A significant width to the iron line was not required, and therefore it was taken to be narrow $(\sigma=0.2 \mathrm{keV})$. No significant measurements of a steepening in the power law at high energies was detected. A Compton reflection component was not required to fit the data with $\Omega / 2 \pi \leq 0.05$.

A bright X-ray transient 2.5 south-west to the nucleus of Cen A, 1RXH J132519.8-430312, was detected by Steinle et al. (2000) during ROSAT HRI observations in July 1995. Parallel multi-wavelength observations from radio to $\gamma$-ray of Cen A were made (Steinle et al. 1999) and no counterpart was found in the optical images. Unfortunately, no spectral information is available from the observations, and only indirect information can be derived from the BATSE and OSSE instruments, which provide evidence that the transient emits mainly at soft X-rays. Steinle et al. (2000) suggested the transient could be an X-ray binary located in Cen A. Assuming a power-law spectrum with photon index 1.5 and a $N_{\mathrm{H}}=810^{20} \mathrm{~cm}^{-2}$, and taking the ROSAT HRI average count rate of 0.033 counts s $^{-1}$ obtained by Steinle et al. (2000), we estimate a RXTE PCA count rate of 0.36 counts s$^{-1} \mathrm{PCU}^{-1}$ (using the PIMMS tool supported 


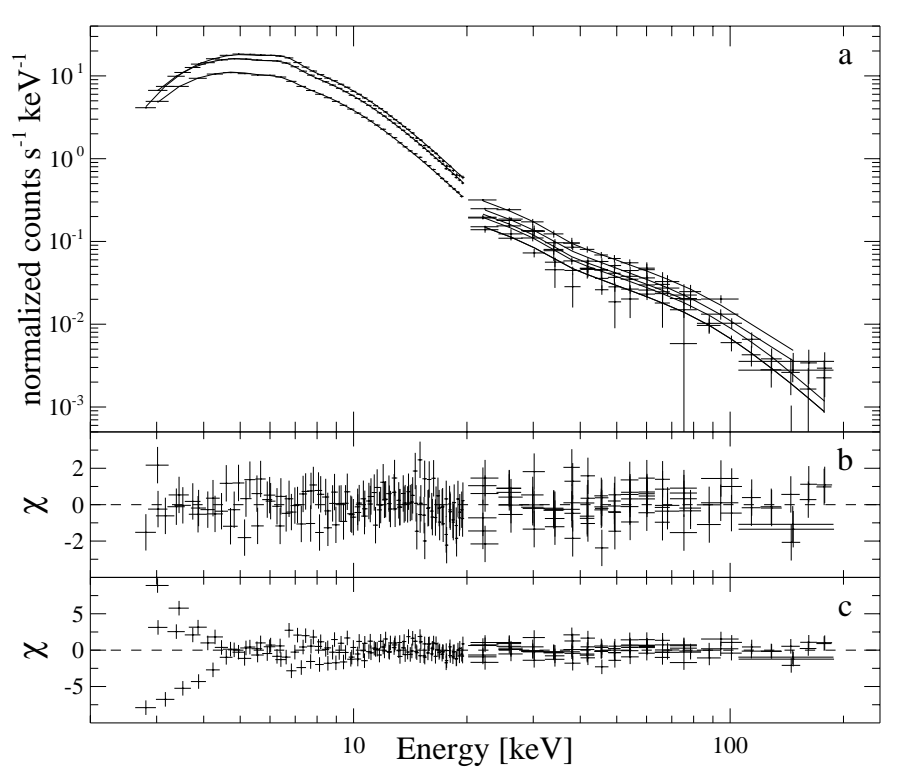

Fig. 2. a) Best fit joined spectrum for an absorbed power law plus Gaussian emission line with $\sigma=0.2 \mathrm{keV}$. See Table 2, joined spectrum, for the exact values of the fit parameters. See text for explanations. b) Residuals to the fit in terms of $\sigma$ with error bars of size one. c) Residuals to the fit with coupled spectral parameter for the three RXTE observations

by HEASARC). This represents $<2 \%$ of the measured Cen A PCA count rate, and therefore no significant contribution of the transient source (if it is supposed to be turned on) on the RXTE observations is expected. This is also confirmed by the spectral behavior of the data, where no excess softness is presented in the spectral fit (see Fig. 2).

\subsection{Timing analysis}

Variability is one of the well known features of Cen A and is observed in all wavelength regimes from radio to $\gamma$-rays (Abraham et al. 1982; Turner et al. 1992; Terrell 1986; Bond et al. 1996; Kinzer et al. 1995). At X-ray wavelengths, Cen A is known to be highly variable on time scales of years, months, and days (Jourdain et al. 1993; Baity et al. 1981). This variability can be split in two main components (Jourdain et al. 1993): (1) long-term trends which last for years and define the state of the source and (2) short-term flares of a few days which are superposed on the long-term component.

\subsubsection{Short term variability}

To characterize the short-term variability of Cen A during the PCA/HEXTE observations we performed a standard $\chi^{2}$-test for the light curves on different time scales. A reduced $\chi_{\nu}^{2}$ value was computed for each stream of data to

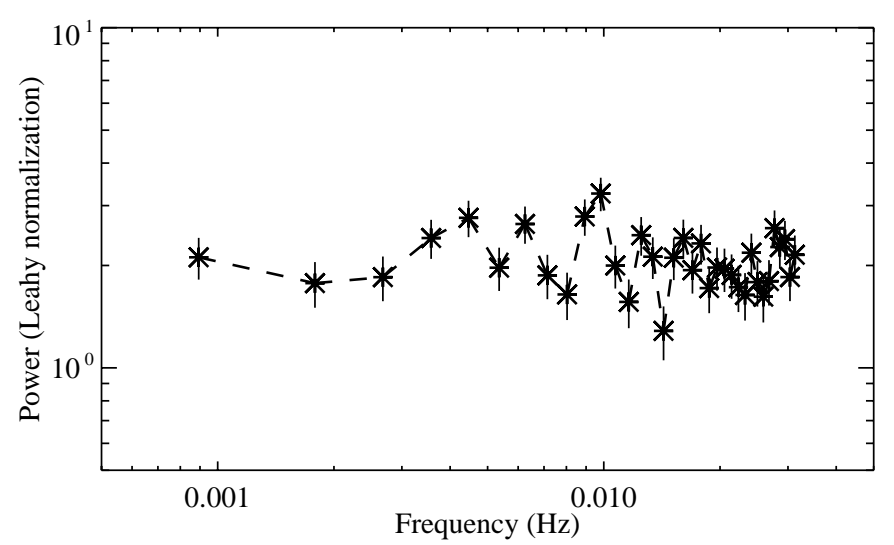

Fig. 3. Averaged power spectral density (PSD) in Leahy et al. (1983) normalization of the PCA background subtracted source data. A PSD value of 2 corresponds to pure Poisson noise

test for variability against the null hypothesis that the flux remains constant:

$\chi_{\nu}^{2}=\frac{1}{\nu} \sum_{i=1}^{n} \frac{\left(c_{i}-\langle c\rangle\right)^{2}}{\sigma_{i}^{2}}$

where $c_{i}$ is the observed count rate at each time interval $i, \sigma_{i}$ the respective Poisson error, $\langle c\rangle$ the mean count rate during the observation, $n$ is the number of time bins, and $\nu=n-1$ is the number of degrees of freedom. For a non variable source we expected $\chi_{\nu}^{2} \sim 1$. No variability within the measurement errors over timescales of days or less was detected for all the pointing observations of RXTE. Variations of $10 \%$ in the mean flux were detected over timescales of $\sim 5 \mathrm{~d}$ during observation 2 (see Fig. 1, middle panel).

Fourier techniques were also used for the analysis of the long 1998 observation. An average power spectral density (PSD) using time segments of $\sim 22$ min duration was calculated. This duration was the longest time span for which enough time segments without gaps were available to compute a PSD. The analysis was performed for the PCA background subtracted data from 4 to $40 \mathrm{keV}$, where the data is source dominated. No excess variability above the Poisson level was seen (see Fig. 3), confirming the result of the $\chi^{2}$ test in this frequency.

To compare the Cen A variability with previous studies of Seyfert Galaxies (Markowitz \& Edelson 2000; Turner et al. 1997b; Nandra et al. 1997) we extracted the $2-10 \mathrm{keV}$ light curve of the 1998 monitoring campaign and computed the normalized "excess variance", $\sigma_{\text {rms }}^{2}$ (for a detailed discussion of the definition see Turner et al. 1999; Nandra et al. 1997):

$\sigma_{\mathrm{rms}}^{2}=\frac{1}{n\langle c\rangle^{2}} \sum_{i=1}^{n}\left[\left(c_{i}-\langle c\rangle\right)^{2}-\sigma_{i}^{2}\right]$.

We find $\sigma_{\mathrm{rms}}^{2}=4.3(9) 10^{-3}$. Previous studies have found a clear decrease of $\sigma_{\mathrm{rms}}^{2}$ with the intrinsic X-ray luminosity of both Seyfert 1 and Seyfert 2 galaxies (consistent also with the time-domain results of König et al. 1997, and 


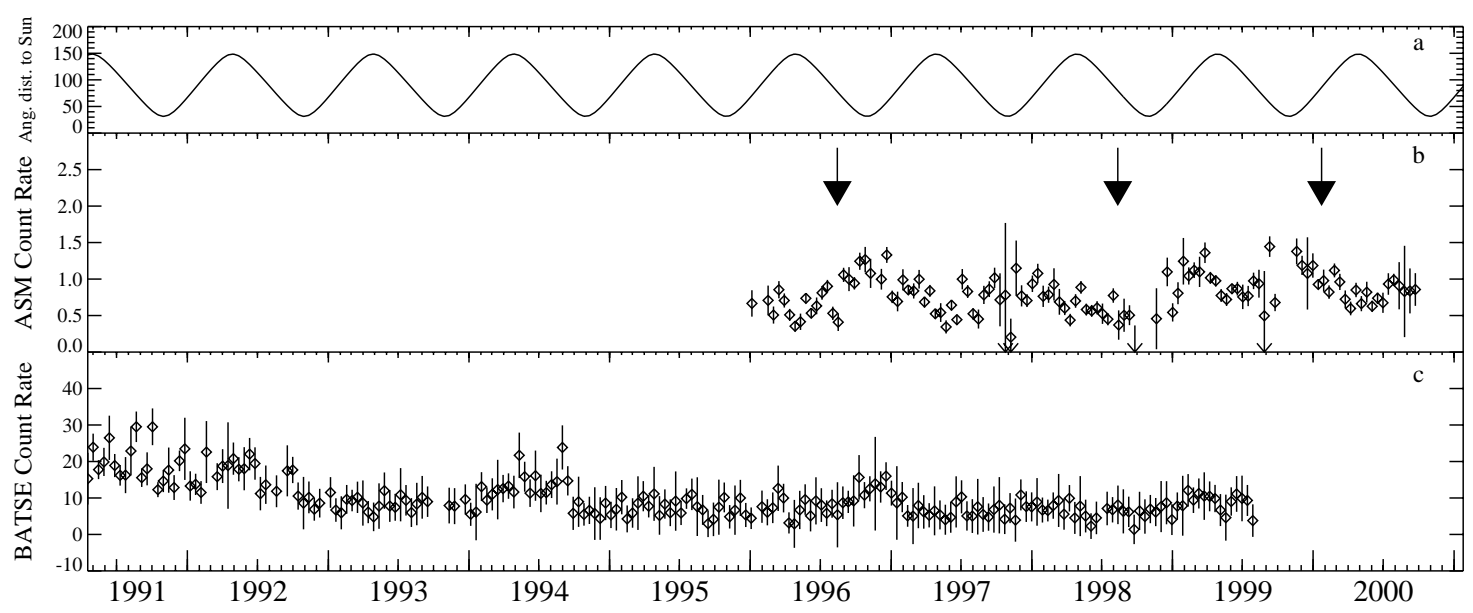

Fig. 4. a) Angular distance between the Sun and Cen A. b) ASM counting rate for Cen A from the beginning of the RXTE measurements, rebinned in $14 \mathrm{~d}$ bins. The RXTE PCA/HEXTE monitoring campaigns are indicated by the arrow. c) BATSE counting rate for Cen A from the beginning of the CGRO measurements, rebinned in $14 \mathrm{~d}$ bins

with Green et al. 1993). Our result for Cen A is a factor of $\sim 10$ smaller than that expected from the Seyfert correlations (Turner et al. 1997b; Nandra et al. 1997) for the intrinsic (i.e., unabsorbed) X-ray luminosity of Cen A $\left(\log L_{\mathrm{X}, 2-10 \mathrm{keV}} \simeq 41.7 \mathrm{cgs}\right)$. Such a low $\sigma_{\text {rms }}^{2}$ is typically only expected for Seyfert 2's with $\log L_{\mathrm{X}} \sim 43 \mathrm{cgs}$ (see Turner et al. 1997b, Fig. 2), although the scatter in these data is large. We conclude that the short term variability of Cen A is on the low side of that observed in comparable Seyfert galaxies.

\subsubsection{Long term variability}

To study the long-term variability of Cen A, and to place our pointed observations within the context of the overall behavior of the source, we used data from the RXTE ASM and CGRO BATSE all sky monitors (see Fig. 4).

Figure 4b indicates apparent fluctuations in the ASM flux at the end of each year coinciding with the closest angular separation between the sun to Cen A (Fig. 4a). This variability is due to scattering of solar X-rays off the ASM collimators onto the ASM detectors and is not due to source flux variations (Remillard, priv. comm.).

The BATSE count rate is shown in Fig. 4c and does not display the variability seen in the ASM. Thus, we conclude that the Cen A flux appears to have varied by less than a factor of two during the four years of the RXTE mission. BATSE data extending back to 1991 indicate that the same conclusion can be drawn back to at least mid-1992. Cen A was a factor of two brighter in the BATSE data from mid-1991 to mid-1992.

\section{Discussion and conclusions}

We have presented analysis of three RXTE monitoring campaigns of Cen A, totaling $\sim 110 \mathrm{ks}$. This analysis reveals no significant short-term temporal variability on timescales from weeks or less, and a relatively simple spectrum in the $2-200 \mathrm{keV}$ range. The best fit spectral model consists of a heavily absorbed primary flux and a narrow emission line from cool iron. The iron line flux remained stable at $\sim 4.510^{-4}$ photons $\mathrm{cm}^{-2} \mathrm{~s}^{-1} \mathrm{keV}^{-1}$ for unabsorbed flux and $\sim 5.310^{-4}$ photons $\mathrm{cm}^{-2} \mathrm{~s}^{-1} \mathrm{keV}^{-1}$ for absorbed flux.

The $2-10 \mathrm{keV}$ flux was the same in 1996 and 1998, and increased by $60 \%$ in 2000 . This implies that the line emitting region is not very close (greater than light days?) to the region containing the primary flux generation. This is consistent with the lack of a significant reflection component contribution to the overall flux. We can conclude that the $\mathrm{Fe}$ line does not originate close to the central X-ray source, although a strong Fe line is observable. Possible sources of the Fe line flux would then be a molecular torus surrounding the source (similar to Seyfert 2 galaxies) or the dust lane. To test the latter hypothesis we have used a Monte Carlo code to compute the iron line flux emerging from the back of a slab of neutral material with $N_{\mathrm{H}}=710^{22} \mathrm{~cm}^{-2}$ that is irradiated by the unattenuated continuum spectrum of Cen A. The equivalent width of the line resulting from the absorbing material is $<30 \mathrm{eV}$ so that most of the contributing $\mathrm{Fe}$ line photons do not come from the absorbing region. In agreement with Grandi et al. (2000) and Woźniak et al. (1998) this leaves us with the conclusion that the Fe line originates within material of a large column outside of the line of sight, for example in the postulated molecular torus. Our conclusion is also confirmed by the presence of no correlation between the variability behavior of $N_{\mathrm{H}}$ and the iron line flux, since $N_{\mathrm{H}}$ varied with observation and the line flux does not. The $N_{\mathrm{H}}$ could originate at the edge of the accretion disk and thus its variability might be due to small changes in the disk structure, while the iron line material lies outside the line of sight.

The lack of a reflection component in radio-loud active galaxies poses a problem for unification models of AGN. There are several ways in which a reflection-free AGN spectrum could be produced. First, the reflecting 
cold material could be removed from the inner regions by being "swept up" by the radio jet. For Cen A, there are indications that such a mechanism might be at work. In a recent interpretation of Hubble Space Telescope images taken with WFPC2 and NICMOS, Marconi et al. (2000) notice an evacuated channel produced by the jet between the nucleus and the first bright knot of the jet. A second possibility might be that the inner regions of the accretion disks of radio-loud AGN are not neutral, but strongly ionized. The source for the ionization could be radiation of the radio-jet, or the accretion disk structure could deviate from that of a simple accretion disk, and be, e.g., advection dominated (the latter is improbable, however, because of to the appreciable X-ray luminosity of the source). Lastly, it could also be possible that the production site of the primary X-rays is not within an accretion disk but within the base of the jet itself, such that the covering factor of any cold material present close to the central black hole is small. Observationally, this last interpretation has been advocated by Kinzer et al. (1995) in the analysis of CGRO OSSE data from Cen A, where the energy dependence of the high energy cutoff of the power-law with source flux is interpreted in terms of a changing maximum energy of a nonthermal electron distribution that would be expected in the jet.

Acknowledgements. We acknowledge the RXTE Science Operations Center staff providing the observations and the Guest Observer Facility for providing support in analyzing them. The research has been partially financed by la Caixa/DAAD grant A/98/19182, by NASA grant NAS530720 , by international NSF travel grant INT-9815741, and by a travel grant from the DAAD. SB and JW acknowledge the hospitality and financial support of the Center for Astrophysics and Space Sciences of the University of California at San Diego. $\mathrm{RR}$ and WH similarly acknowledge the friendly atmosphere of the Institut für Astronomie und Astrophysik of the University of Tübingen. CSR appreciates support from Hubble Fellowship grant HF-01113.01-98A. This grant was awarded by the Space Telescope Institute, which is operated by the Association of Universities for Research in Astronomy, Inc., for NASA under contract NAS 5-26555.

\section{References}

Abraham, Z., Kaufmann, P., \& Botti, L. 1982, AJ, 87, 532

Arnaud, K. A. 1996, in Astronomical Data Analysis Software and Systems V, ed. G. H. Jacoby, \& J. Barnes, ASP Conf. Ser. 101 (San Francisco: Astron. Soc. Pacific), 17

Baity, W. A., Rothschild, R. E., Lingenfelter, R. E., et al. 1981, ApJ, 244, 429

Bond, I. A., Ballet, J., Denis, M., et al. 1996, A\&A, 307, 708

Dermer, C. D., \& Gehrels, N. 1995, ApJ, 447, 103

Döbereiner, S., Junkes, N., Wagner, S. J., et al. 1996, ApJ, 470, L15

Ebneter, K., \& Balick, B. 1983, PASP, 95, 675

Fanaroff, B. L., \& Riley, J. M. 1974, MNRAS, 167, 31

Feigelson, E. D., Schreier, E. J., Delvaille, J. P., et al. 1981, ApJ, 251, 31

Grandi, P., Urry, C. M., Maraschi, L., et al. 2000, Adv. Space Res., 25, 485
Green, A. R., McHardy, I. M., \& Lehto, H. J. 1993, MNRAS, 265,664

Hui, X., Ford, H. C., Ciardullo, R., \& Jacoby, G. H. 1993, ApJ, 414, 463

Israel, F. P. 1998, A\&AR, 8, 237

Jahoda, K., Swank, J. H., Giles, A. B., et al. 1996, in EUV, XRay, and Gamma-Ray Instrumentation for Astronomy VII, ed. O. H. Siegmund \& M. A. Gummin, Proc. SPIE 2808 (Bellingham, WA: SPIE), 59

Jourdain, E., Bassani, L., Roques, J. P., et al. 1993, ApJ, 412, 586

Kinzer, R. L., Johnson, W. N., Dermer, C. D., et al. 1995, ApJ, 449, 105

König, M., Staubert, R., \& Wilms, J. 1997, A\&A, 326, L25

Kraft, R. P., Forman, W., Jones, C., et al. 2000, ApJ, 531, L9

Krolik, J. H. 1999, Active Galactic Nuclei (Princeton, NJ: Princeton University Press)

Leahy, D. A., Darbro, W., Elsner, R. F., et al. 1983, ApJ, 266, 160

Levine, A. M., Bradt, H., Cui, W., et al. 1996, ApJ, 469, L33

Levine, A. M., Bradt, H. V., Enevoldsen, A., et al. 2000, BAAS, 32,1260

Magdziarz, P., \& Zdziarski, A. A. 1995, MNRAS, 273, 837

Marconi, A., Schreier, E. J., Koekemoer, A., et al. 2000, ApJ, 528, 276

Markowitz, A., \& Edelson, R. 2000, in Rossi2000: Astrophysics with the Rossi X-ray Timing Explorer, March 22-24, 2000 at NASA's Goddard Space Flight Center, Greenbelt, MD USA, E38

Morganti, R., Fosbury, R. A. E., Hook, R. N., Robindon, A., \& Tsvetanov, Z. 1992, MNRAS, 256, 1

Morganti, R., Killeen, N. E. B., Ekers, R. D., \& Oosterloo, T. A. 1999, MNRAS, 307, 750

Morrison, R., \& McCammon, D. 1983, ApJ, 270, 119

Nandra, K., George, I. M., Mushotzky, R. F., Turner, T. J., \& Yaqoob, T. 1997, ApJ, 476, 70

Rothschild, R. E., Band, D. L., Blanco, P. R., et al. 1999, ApJ, 510,651

Rothschild, R. E., Blanco, P. R., Gruber, D. E., et al. 1998, ApJ, 496, 538

Schreier, E. J., Feigelson, E., Delvaille, J., et al. 1979, ApJ, 234, L39

Smith, D. A., \& Done, C. 1996, MNRAS, 280, 355

Steinle, H., Bennett, K., Bloemen, H., et al. 1998, A\&A, 330, 97

Steinle, H., Bonnell, J., Kinzer, R. L., et al. 1999, Adv. Space Res., 23, 911

Steinle, H., Dennerl, K., \& Englhauser, J. 2000, A\&A, 357, L57

Terrell, J. 1986, ApJ, 300, 669

Turner, P. C., Forrest, W. J., Pipher, J. L., \& Shure, M. A. 1992, ApJ, 393, 648

Turner, T. J., George, I. M., Mushotzky, R. F., \& Nandra, K. 1997a, ApJ, 475, 118

Turner, T. J., George, I. M., Nandra, K., \& Mushotzky, R. F. 1997b, ApJS, 113, 23

Turner, T. J., George, I. M., Nandra, K., \& Turcan, D. 1999, ApJ, 524, 667

Wilms, J., Nowak, M. A., Dove, J. B., Fender, R. P., \& di Matteo, T. 1999, ApJ, 522, 460

Woźniak, P. R., Zdziarski, A. A., Smith, D., Madejski, G. M., \& Johnson, W. N. 1998, MNRAS, 299, 449 